\title{
Cationic Polymerization of $p$-Methoxystyrene by the Triphenylmethyl Bromide/Iodine Initiating System: Formation of Long-Lived Polymers in Polar Solvent
}

\author{
Günther Heublein, Stefan Spange, Mitsuo Sawamoto, ${ }^{*}$ \\ and Toshinobu HIgASHIMURA* \\ Department of Chemistry, Friedrich-Schiller-University, \\ DDR-6900 Jena, German Democratic Republic \\ * Department of Polymer Chemistry, Faculty of Engineering, \\ Kyoto University, Kyoto 606, Japan
}

(Received April 15, 1985)

\begin{abstract}
Triphenylmethyl bromide in conjunction with iodine $\left(\mathrm{Ph}_{3} \mathrm{CBr} / \mathrm{I}_{2}\right.$ initiator) induced cationic polymerization of $p$-methoxystyrene (pMOS) in 1,2-dichloroethane at $-15^{\circ} \mathrm{C}$ to yield long-lived (living-like) polymers. The long lifetime of the polymers was indicated by a linear progressive increase of their number-average molecular weight $\left(M_{n}\right)$ with pMOS conversion and the further increase of $M_{n}$ on the addition of a fresh pMOS feed to a completely polymerized solution. $\mathrm{UV} /$ visible spectoscopic analysis showed that iodine promotes the dissociation of $\mathrm{Ph}_{3} \mathrm{CBr}$ into the triphenylmethyl cation which in turn initiates the polymerization of pMOS. A mixture of triphenylmethyl thiocyanate $\left(\mathrm{Ph}_{3} \mathrm{CSCN}\right)$ and iodine also acted as an initiator for pMOS polymerization; the overall polymerization rate decreased in the following order: $\mathrm{Ph}_{3} \mathrm{CBr} / \mathrm{I}_{2}>$ $\mathrm{Ph}_{3} \mathrm{CSCN} / \mathrm{I}_{2}>\mathrm{I}_{2}$.

KEY WORDS Cationic Polymerization / Living Polymers / Long-Lived Polymers / Triphenylmethyl Bromide / Triphenylmethyl Thiocyanate / Iodine / $p$-Methoxystyrene / Triphenylmethyl Cation / Initiation /
\end{abstract}

The cationic polymerization of $p$-methoxystyrene (pMOS) initiated by iodine involves a long-lived propagating species that is favorably formed at low temperatures in nonpolar media (e.g., carbon tetrachloride) ${ }^{1,2}$ The reaction exhibits characteristics reminiscent of "living" polymerization; i.e., a progressive increase in the number-average molecular weight $\left(M_{n}\right)$ of the product polymers with increasing monomer conversion, its further raise on the addition of a fresh pMOS feed to a completely polymerized reaction mixture, and the formation of pMOS-isobutyl vinyl ether block copolymers. $^{2}$ These features indicate that chain transfer and termination reactions are considerably suppressed in the pMOS/iodine system, ${ }^{3}$ where the stable pMOS growing end is associated with the nucleophilic iodide counteranion. We have theorized ${ }^{4}$ that the strong interaction of the counteranion with the propagating end is of key importance to suppress the side reactions deleterious to living polymerization. Such a strong cation-anion interaction in the growing species also occurs in the polymerization of vinyl ethers by a mixture of hydrogen iodide and iodine $\left(\mathrm{HI} / \mathrm{I}_{2}\right.$ initiator) where perfectly living, monodisperse polymers are produced. ${ }^{5,6}$

Among initiators for cationic vinyl polymerization, molecular iodine and $\mathrm{HI} / \mathrm{I}_{2}$ initiator are known to yield living/long-lived propagating species associated with a strongly interacting counteranion. ${ }^{4}$ It occurred to us that counteranions similar in nature to the iodide anion might form from a suitable carbocation source (RX) combined with 
iodine, as illustrated in eq 1 :

$$
\begin{aligned}
\mathrm{RX}+\mathrm{I}_{2} & \rightleftharpoons\left[\mathrm{R}-\mathrm{X} \cdots \mathrm{I}_{2}\right] \\
& \rightleftharpoons \mathrm{R}^{\oplus} \cdot \mathrm{XI}_{2}^{\ominus} \stackrel{\mathrm{pMOS}}{ } \text { Polymers }
\end{aligned}
$$

where $\mathrm{R}$ and $\mathrm{X}$ represent, respectively, the triphenylmethyl group and a leaving group (e.g., halogen). If living/long-lived polymers are obtained with $\mathrm{XI}_{2}^{\ominus}$ as counteranion, the $\mathrm{RX} / \mathrm{I}_{2}$ initiator will be of importance not only as a new entry to the initiators for living polymerization of vinyl monomers but in generalizing our priciple of living cationic polymerization based on the strong interaction between the growing carbocation and the counteranion.

In this study, we employed triphenylmethyl bromide $\left(\mathrm{Ph}_{3} \mathrm{CBr} ; \mathrm{X}=\mathrm{Br}\right)$ and triphenylmethyl thiocyanate $\left(\mathrm{Ph}_{3} \mathrm{CSCN} ; \mathrm{X}=\mathrm{SCN}\right)$ as $\mathrm{RX}$ in eq 1 , and polymerized pMOS using these $\mathrm{RX} / \mathrm{I}_{2}$ initiating systems. Triphenylmethyl cation salts having a complex metalhalide counteranion (e.g., $\mathrm{SbF}_{6}^{\ominus}$ ) are well known and thoroughly studied as initiators for cationic polymerization of pMOS and vinyl ethers. ${ }^{7}$ Recently, two of $\mathrm{us}^{8}$ found that a series of triphenylmethyl halide/electron acceptor pairs (e.g., $\mathrm{Ph}_{3} \mathrm{CBr} /$ chloranil) can also polymerize pMOS in polar media. None of these triphenylmethyl cation-based initiators, however, successfully lead to living/long-lived poly(pMOS). We wish to report that the $\mathrm{Ph}_{3} \mathrm{CBr} / \mathrm{I}_{2}$ initiating system effectively polymerizes pMOS in 1,2-dichloroethane (DCE) to give long-lived polymers. The polar solvent was required for the formation of long-lived poly (pMOS), rather than nonpolar media better suited for the iodine-initiated polymerization. ${ }^{1,2}$

\section{EXPERIMENTAL}

\section{Meterials}

Triphenylmethyl bromide was synthesized from triphenylmethanol and acetyl bromide (freshly distilled) in dry benzene by the stan- dard method. ${ }^{9}$ The crude product was recrystallized from a benzene/hexane mixture $(1: 10 \mathrm{v} / \mathrm{v})$. Triphenylmethyl thiocyanate was obtained by the reaction of triphenylmethyl chloride or bromide with potassium thiocyanate in carefully purified dry acetone. ${ }^{10}$ Recrystallization from acetone gave the pure thiocyanate as white needles. Iodine was sublimed over potassium iodide and stored under argon in the dark.

pMOS was prepared from $p$-methoxybenzaldehyde by the Grignard reaction with methylmagnesium bromide. ${ }^{11}$ It was purified by vacuum distillation over calcium hydride and stored in a refrigerator. DCE as the polymerization solvent was purified by the usual method and distilled twice successively over phosphorus pentoxide and calcium hydride just before use.

\section{Procedures}

Polymerizations were carried out in DCE at $-15^{\circ} \mathrm{C}$ under dry argon in a baked glass tube equipped with a three-way stopcock. The reaction was initiated by adding an initiator solution into a monomer solution and terminated with prechilled ammoniacal methanol. The initiator solution was prepared by dissolving iodine and triphenylmethyl bromide or thiocyanate in DCE at room temperature and then cooled to the polymerization temperature before mixed with the monomer. For some runs iodine alone was used as an initiator.

The conversion of pMOS was determined either by gravimetry of the isolated polymers (see below) or by ultraviolet (UV) spectroscopy monitoring a decrease in the pMOS absorption at $295 \mathrm{~nm}$; the values by the two methods were in good agreement within $5 \%$, indicating the absence of methanol-soluble oligomers in the product.

The produced poly(pMOS) was precipitated into methanol, filtered off, washed exhaustively with methanol, and dried in vacuo below $20^{\circ} \mathrm{C}$. The molecular weight distribution (MWD) of the polymers was measured by size exclusion 
chromatography (SEC) in tetrahydrofuran

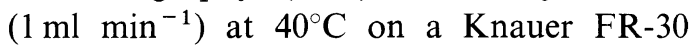
chromatograph equipped with 7 silica gel columns (Merck. LiChrospher Si 4000, 1000, 500, 300,100 , and $60(\times 2) ; 4 \mathrm{~mm}$ i.d. $\times 250 \mathrm{~mm}$ each) and a UV detector operating at $254 \mathrm{~nm}$. A $0.5 \mathrm{w} / \mathrm{v} \%$ solution of the polymer sample was injected. The $M_{n}$ and $M_{w} / M_{n}$ ratio were calculated from SEC eluograms on the basis of a polystyrene calibration.

$\mathrm{UV} /$ visible spectra were recorded in DCE at $20^{\circ} \mathrm{C}$ on a SPECORD spectrophotometer (VEB Carl Zeiss, Jena), as previously described. ${ }^{12}$

\section{RESULTS AND DISCUSSION}

pMOS Polymerization by Triphenylmethyl Compound/Iodine Initiating Systems

pMOS was polymerized in DCE at $-15^{\circ} \mathrm{C}$ by $\mathrm{Ph}_{3} \mathrm{CBr}$ or $\mathrm{Ph}_{3} \mathrm{CSCN}$ coupled with an equimolar amount of iodine. For comparison iodine alone was also used otherwise under the same conditions. Although neither $\mathrm{Ph}_{3} \mathrm{CBr}$ nor $\mathrm{Ph}_{3} \mathrm{CSCN}$ per se could induce pMOS polymerization, when coupled with iodine both triphenylmethyl compounds polymerized pMOS almost quantitatively. The overall polymerization rates by $\mathrm{Ph}_{3} \mathrm{CBr} / \mathrm{I}_{2}$ and $\mathrm{Ph}_{3} \mathrm{CSCN} /$ $\mathrm{I}_{2}$ initiators clearly exceeded that by iodine alone; reaction time for $95 \%$ conversion: $\mathrm{Ph}_{3} \mathrm{CBr} / \mathrm{I}_{2}(10 \mathrm{~min})>\mathrm{Ph}_{3} \mathrm{CSCN} / \mathrm{I}_{2}(60 \mathrm{~min})>$ $\mathrm{I}_{2}(180 \mathrm{~min})$.

Another dependence on the initiators was found in the MWD of the produced polymers (Figure 1). $\mathrm{Ph}_{3} \mathrm{CBr} / \mathrm{I}_{2}$ and $\mathrm{Ph}_{3} \mathrm{CSCN} / \mathrm{I}_{2}$ initiators led to polymers with a unimodal MWD; iodine alone also gave a broad, unimodal MWD as already reported. ${ }^{1,2}$ Importantly, the MWD for $\mathrm{Ph}_{3} \mathrm{CBr} / \mathrm{I}_{2}$ was considerably narrower than those for $\mathrm{Ph}_{3} \mathrm{CSCN} / \mathrm{I}_{2}$ and iodine.

These dependences of the reaction rate and the MWD of polymers show that the triphenylmethyl salt-based initiators effect a pMOS polymerization clearly differing from

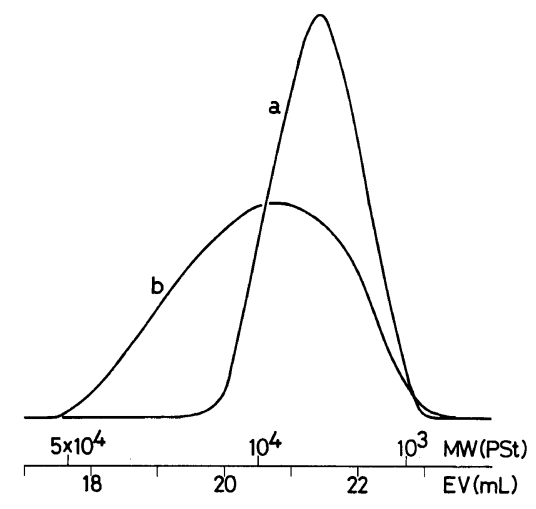

Figure 1. MWD of poly(pMOS) obtained in DCE at $-15^{\circ} \mathrm{C}:[\mathrm{M}]_{0}=0.40 \mathrm{M}$; conversion $>95 \%$.

(a) $\left[\mathrm{Ph}_{3} \mathrm{CBr}\right]_{0}=\left[\mathrm{I}_{2}\right]_{0}=5.0 \mathrm{mM}$;

(b) $\left[\mathrm{Ph}_{3} \mathrm{CSCN}\right]_{0}=\left[\mathrm{I}_{2}\right]_{0}=5.0 \mathrm{mM}$.

that by iodine alone. Since the polymerizations by $\mathrm{Ph}_{3} \mathrm{CBr} / \mathrm{I}_{2}$ and $\mathrm{Ph}_{3} \mathrm{CSCN} / \mathrm{I}_{2}$ also considerably differ from each other, the counteranions $\left(\mathrm{Br}^{\ominus}\right.$ and $\left.\mathrm{SCN}^{\ominus}\right)$ of the triphenylmethyl salts should be involved in the initiation/propagation processes (see below).

Formation of Long-Lived Polymers by $\mathrm{Ph}_{3} \mathrm{CBr} /$ $\mathrm{I}_{2}$ Initiator

In view of the fairly narrow MWD $\left(M_{w} /\right.$ $\left.M_{n}=1.66\right)$ of poly(pMOS) obtained by $\mathrm{Ph}_{3} \mathrm{CBr} / \mathrm{I}_{2}$ initiator (Figure 1), living/longlived polymers may form in the pMOS polymerization by this combined initiator. The most important diagnostic characteristic of living polymers is that they can resume propagation when an additional feed of monomer is supplied to a polymerization solution, leading to an increase in their $M_{n}$ with increasing number of the monomer addition. We then examined the $\mathrm{Ph}_{3} \mathrm{CBr} / \mathrm{I}_{2}$-initiated polymerization of pMOS more in detail from this viewpoint (so-called monomer-addition experiments ${ }^{4}$ ).

In DCE at $-15^{\circ} \mathrm{C}$ pMOS was polymerized by $\mathrm{Ph}_{3} \mathrm{CBr} / \mathrm{I}_{2}$ initiator, and a small amount of fresh pMOS (neat) was repeatedly added to the reaction mixture when the previously supplied monomer had completely depleted. Figure 2 


\section{G. HEUBLEIN et al.}

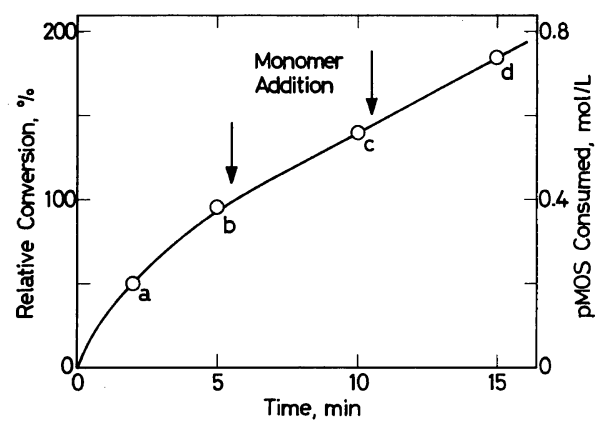

Figure 2. Time-conversion curve for a monomeraddition experiment in the pMOS polymerization by $\mathrm{Ph}_{3} \mathrm{CBr} / \mathrm{I}_{2}$ initiator in DCE at $-15^{\circ} \mathrm{C}:[\mathrm{M}]_{0}=0.40 \mathrm{M}$; $\left[\mathrm{Ph}_{3} \mathrm{CBr}\right]_{0}=\left[\mathrm{I}_{2}\right]_{0}=5.0 \mathrm{mM}$. At each monomer-addition step (indicated by the arrow), neat pMOS, one half the molar quantity of the initial charge, was added into the reaction mixture.

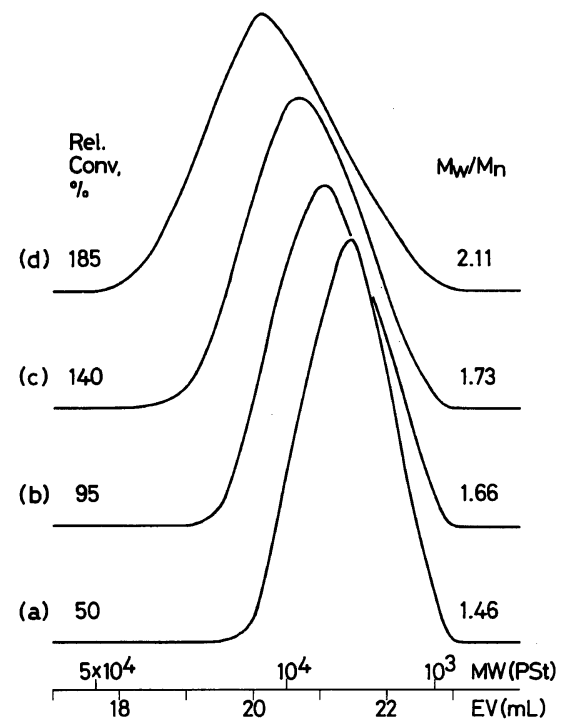

Figure 3. MWD of poly(pMOS) obtained in the monomer-addition experiment described in Figure 2. As to the sample codes and reaction conditions, see the caption for Figure 2.

shows the time-conversion curve for such a monomer-addition experiment. After each monomer addition, the added pMOS polymerized nearly quantitatively without an apparent change in polymerization rate, indicating the absence of termination.

Figure 3 illustrates the MWD of a series of polymer samples obtained in the monomer-

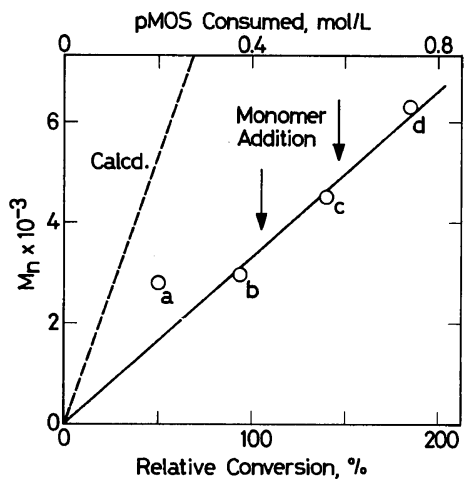

Figure 4. $M_{n}$-conversion profile for poly(pMOS) obtained in the monomer-addition experiment described in Figure 2. As to the sample codes and reaction conditions see the caption for Figure 2. The broken line indicates the calculated values based on the quantitative initiation of $\mathrm{Ph}_{3} \mathrm{CBr} / \mathrm{I}_{2}$.

addition experiment. In the first-stage polymerization (Figure 3, a and b) the MWD clearly shifted toward high molecular weight with increasing conversion. A further increase in polymer molecular weight was observed in the second- and third-stage polymerizations (Figure 3, c and d, respectively), in which a new pMOS feed was added to the reaction mixture. The monomer addition also resulted in a considerable broadening of the MWD, although the peak molecular weight clearly increased at each stage.

The increase in polymer molecular weight on the repeated monomer-addition is depicted more quantitatively in the $M_{n}$-conversion plots in Figure 4 . The $M_{n}$ was nearly proportional to pMOS conversion (or the total amount of pMOS consumed), and the linear rise of $M_{n}$ continued after the monomer addition. All these facts show the formation of long-lived polymers in the polymerization initiated by $\mathrm{Ph}_{3} \mathrm{CBr} / \mathrm{I}_{2}$ in DCE. Iodine alone, in contrast, does not yield such long-lived poly(pMOS) in polar media. ${ }^{1,2}$

In spite of the linear increase in polymer molecular weight, however, the polymerization by $\mathrm{Ph}_{3} \mathrm{CBr} / \mathrm{I}_{2}$ cannot be referred to as a "living" process, because of the consecutive 
broadening of the MWD of polymers upon monomer addition (Figure 3, c and d). Thus, the propagating species does have a long lifetime, but may undergo chain transfer to some extent. Another indication of the occurrence of chain transfer is that the observed $M_{n}$ 's (by SEC) are smaller than the calculated values (Figure 4, broken line) based on the quantitative initiation by $\mathrm{Ph}_{3} \mathrm{CBr} / \mathrm{I}_{2}$.

\section{Polymerization Mechanism: Dissociation of}

Triphenylmethyl Bromide Assisted by Iodine

The clear difference between the polymerizations by $\mathrm{Ph}_{3} \mathrm{CBr} / \mathrm{I}_{2}$ initiator and by iodine alone, described above, shows a cooperative action of $\mathrm{Ph}_{3} \mathrm{CBr}$ and iodine in the initiation/ propagation processes. Eq 2 gives the most likely initiation mechanism in which $\mathrm{Ph}_{3} \mathrm{CBr}$

$$
\begin{aligned}
& \mathrm{Ph}_{3} \mathrm{CBr}+\mathrm{I}_{2} \stackrel{\mathrm{DCE}}{\rightleftharpoons}\left[\mathrm{Ph}_{3} \mathrm{C}-\mathrm{Br} \cdots \mathrm{I}_{2}\right] \\
& \rightleftharpoons \mathrm{Ph}_{3} \mathrm{C}^{\oplus} \cdot \mathrm{BrI}_{2} \stackrel{\text { pMOS }}{\rightleftharpoons}
\end{aligned}
$$

Long-lived polymers

dissociates into the triphenylmethyl cation $\left(\mathrm{Ph}_{3} \mathrm{C}^{\oplus}\right)$ with the aid of the electrophilic interaction of iodine on the bromine atom. To find evidence for this mechanism, we studied the interaction between $\mathrm{Ph}_{3} \mathrm{CBr}$ and iodine, either in the presence or absence of pMOS monomer, by UV/visible spectroscopy in DCE at $20^{\circ} \mathrm{C}$. Figure 5 presents typical spectra thus obtained.

$\mathrm{Ph}_{3} \mathrm{CBr}$ alone shows very weak absorptions of the $\mathrm{Ph}_{3} \mathrm{C}^{\oplus}$ cation at 413 and $440 \mathrm{~nm}$ (Figure $5 \mathrm{a})$. The addition of a slightly excess of iodine to the $\mathrm{Ph}_{3} \mathrm{CBr}$ solution led to a nearly 10 -fold increase of the $\mathrm{Ph}_{3} \mathrm{C}^{\oplus}$ cation (Figure 5c). In this spectrum the absorption of iodine ( $c a$. $500 \mathrm{~nm} ; c f$. Figure $5 \mathrm{~b}$ ) is seen overlapping with the $\mathrm{Ph}_{3} \mathrm{C}^{\oplus}$ band.

When the $\mathrm{Ph}_{3} \mathrm{CBr} / \mathrm{I}_{2}$ mixture was added to an excess of pMOS, namely, under the conditions simulating the pMOS polymerization by $\mathrm{Ph}_{3} \mathrm{CBr} / \mathrm{I}_{2}$ initiator, the double peak of

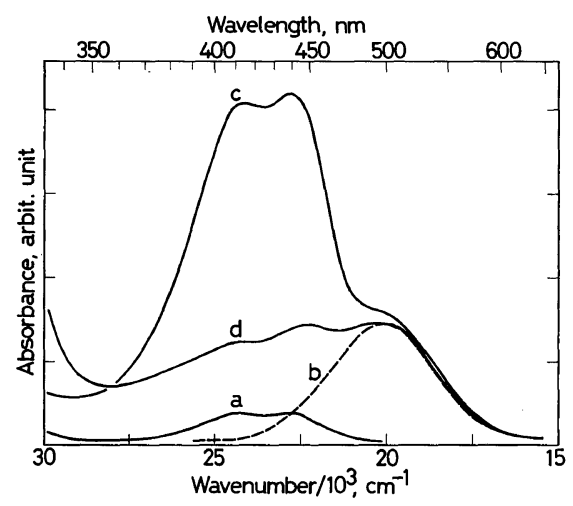

Figure 5. UV/visible spectra in DCE at $+20^{\circ} \mathrm{C}$ (concentrations in $\mathrm{mM}$ ): (a) $\mathrm{Ph}_{3} \mathrm{CBr}(0.30)$; (b) $\mathrm{I}_{2}$ (0.50); (c) $\mathrm{Ph}_{3} \mathrm{CBr} \quad(0.30)+\mathrm{I}_{2} \quad(0.50) ; \quad$ (d) $\mathrm{Ph}_{3} \mathrm{CBr} \quad(0.30)+\mathrm{I}_{2}$ $(0.50)+$ pMOS (8.0). Spectra $\mathrm{c}$ and $\mathrm{d}$ were recorded immediately after mixing all ingredients.

$\mathrm{Ph}_{3} \mathrm{C}^{\oplus}$ was still observable but its intensity decreased by factor of about 3 (Figure 5d). The iodine absorption was detected at $c a$. $500 \mathrm{~nm}$ even in the presence of pMOS, indicating that a part of the added halogen remains intact during the polymerization.

The enhanced formation of the $\mathrm{Ph}_{3} \mathrm{C}^{\oplus}$ cation from $\mathrm{Ph}_{3} \mathrm{CBr}$ in the presence of iodine (Figure 5b) shows that added iodine promotes the dissociation of the triphenylmethyl salt by its electrophilic interaction with the bromine atom; i.e., iodine polarizes the carbon-bromine bond of $\mathrm{Ph}_{3} \mathrm{CBr}$. The resulting $\mathrm{Ph}_{3} \mathrm{C}^{\oplus}$ cation in turn adds across the double bond of pMOS and thereby initiates cationic polymerization of this monomer. The considerable decrease of the $\mathrm{Ph}_{3} \mathrm{C}^{\oplus}$ cation in the presence of pMOS (Figure 5d) supports its efficient addition (initiation) to the vinyl monomer. Thus, the proposed initiation mechanism (eq 2) was experimentally verified, although the rather intense tailings in the region from 340 to $390 \mathrm{~nm}$, due to the absorptions of pMOS $\left(\lambda_{\max }=\right.$ $295 \mathrm{~nm}$ ) and $\mathrm{Ph}_{3} \mathrm{C}^{\oplus}$, hampered us to detect the propagating pMOS cation that would exhibit a spectrum centered at $380 \mathrm{~nm}^{13}$

The iodine-assisted polarization (dissociation) of a carbon-halogen bond, similar to that in the $\mathrm{Ph}_{3} \mathrm{CBr} / \mathrm{I}_{2}$ system, has been 
found in the living polymerization of vinyl ethers by $\mathrm{HI} / \mathrm{I}_{2}$ initiator (eq 3): 5,6

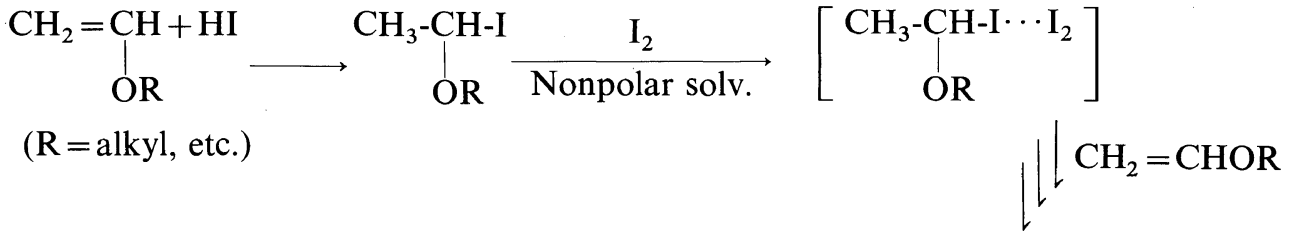

Living polymers
In this initiation process, the carbon-iodine covalent bond is polarized (electrophilically "activated" 6 ) by iodine in nonpolar solvents. ${ }^{14}$ An important difference between $\mathrm{Ph}_{3} \mathrm{CBr} / \mathrm{I}_{2}$ and $\mathrm{HI} / \mathrm{I}_{2}$ initiators, therefore, is that $\mathrm{Ph}_{3} \mathrm{CBr}$ requires a polar solvent (DCE) for its dissociation assisted by iodine.

This study has shown that $\mathrm{Ph}_{3} \mathrm{CBr}$ in conjunction with iodine is not only an effective initiator for the cationic polymerization of pMOS but produces long-lived polymers in DCE. The $\mathrm{Ph}_{3} \mathrm{CBr} / \mathrm{I}_{2}$-initiated polymerization is unique in that long-lived polymers form, first, in a relatively polar solvent (DCE) and, second, with a mixed halide ion $\left(\mathrm{BrI}_{2}{ }^{\ominus}\right)$ as the counteranion. It should be noted that in such polar media, iodine alone results in polymers with a broad MWD via transfer-dominant polymerization. ${ }^{1,2}$ The second point is of importance to expand the scope of initiators (counteranions) for living/long-lived vinyl polymers, which have thus far been confined to iodine and $\mathrm{HI} / \mathrm{I}_{2}$.

\section{REFERENCES AND NOTES}

1. T. Higashimura and O. Kishiro, Polym. J., 9, 87 (1977).

2. T. Higashimura, M. Mitsuhashi, and M. Sawamoto,
Macromolecules, 12, 178 (1979).

3. In a strict sence, this polymerization cannot be referred to as a "perfectly living" process where chain transfer and termination are absent, since the number of polymer chains produced per unit iodine increases with conversion and the product polymers are not monodisperse. ${ }^{1,2}$

4. T. Higashimura and M. Sawamoto, Adv. Polym. Sci., 62, 49 (1984).

5. M. Miyamoto, M. Sawamoto, and T. Higashimura, Macromolecules, 17, 265, 2228 (1984).

6. (a) T. Higashimura, M. Miyamoto, and $M$. Sawamoto, Macromolecules, 18, 611 (1985). (b) M. Sawamoto, M. Miyamoto, and T. Higashimura, Polym. Prepr., Jpn., 33, 1399 (1984).

7. As reviews, see: (a) A Ledwith and D. C. Sherrington, Adv. Polym. Sci., 19, 1 (1975); (b) A. Gandini and H. Cheradame, ibid., 34/35, 1 (1980).

8. G. Heublein and S. Spange, Acta Polym., 33, 509 (1982).

9. W. E. Bachmann, Org. Syn., 23, 100 (1943); Org. Synth. Coll. Vol. 3, 841 (1955).

10. A. Iliceto, A. Fava, and V. Mazzuccato, J. Org. Chem., 25, 1445 (1960).

11. R. Cotrel, G. Sauvet, J. P. Vairon, and P. Sigwalt, Macromolecules, 9, 931 (1976).

12. G. Heublein, S. Spange, and P. Hallpap, Makromol. Chem., 180, 1935 (1979).

13. M. Sawamoto and T. Higashimura, Macromolecules, 11, 328 (1978).

14. We have recently found that the living polymerization of vinyl ethers by $\mathrm{HI} / \mathrm{I}_{2}$ initiator proceeds in not only nonpolar but polar media such as methylene chloride as well: T. Higashimura, T. Enoki, and M. Sawamoto, to be published. 